PROCEEDINGS OF THE

AMERICAN MATHEMATICAL SOCIETY

Volume 135, Number 3, March 2007, Pages 637-648

S 0002-9939(06)08519-4

Article electronically published on August 28, 2006

\title{
HILBERT-SAMUEL FUNCTIONS OF MODULES OVER COHEN-MACAULAY RINGS
}

\author{
SRIKANTH IYENGAR AND TONY J. PUTHENPURAKAL
}

(Communicated by Bernd Ulrich)

\begin{abstract}
For a finitely generated, non-free module $M$ over a CM local ring $(R, \mathfrak{m}, k)$, it is proved that for $n \gg 0$ the length of $\operatorname{Tor}_{1}^{R}\left(M, R / \mathfrak{m}^{n+1}\right)$ is given by a polynomial of degree $\operatorname{dim} R-1$. The vanishing of $\operatorname{Tor}_{i}^{R}\left(M, N / \mathfrak{m}^{n+1} N\right)$ is studied, with a view towards answering the question: If there exists a finitely generated $R$-module $N$ with $\operatorname{dim} N \geq 1$ such that the projective dimension or the injective dimension of $N / \mathfrak{m}^{n+1} N$ is finite, then is $R$ regular? Upper bounds are provided for $n$ beyond which the question has an affirmative answer.
\end{abstract}

\section{INTRODUCTION}

Let $(R, \mathfrak{m}, k)$ denote a local ring with maximal ideal $\mathfrak{m}$ and residue field $k$; in this article local rings are assumed to be Noetherian. Let $M$ be a finite, that is to say, finitely generated, $R$-module.

Recall that the Hilbert-Samuel function of $M$ is the function on the non-negative integers that maps $n$ to $\ell_{R}\left(M / \mathfrak{m}^{n+1} M\right)$, where $\ell_{R}(-)$ denotes length. The HilbertSamuel function is an important invariant of $M$, and has long been a topic of active research. Note that $M / \mathfrak{m}^{n+1} M=\operatorname{Tor}_{0}^{R}\left(M, R / \mathfrak{m}^{n+1}\right)$. This suggests the following line of enquiry: Fix an integer $i \geq 0$ and consider the function

$$
n \mapsto \ell_{R} \operatorname{Tor}_{i}^{R}\left(M, R / \mathfrak{m}^{n+1}\right) \quad \text { for each } n \in \mathbb{N} .
$$

This may be thought of as the ith Hilbert-Samuel function of $M$. For each $i$, this function is given by a polynomial for $n \gg 0$, which we denote $\tau_{i}^{R}(M ; z)$. This result is classical for $i=0$, and the polynomial $\tau_{0}^{R}(M ; z)$ is the Hilbert-Samuel polynomial of $M$. For general $i$, it is due to Kodiyalam [5]; see also Theodorescu [13].

Recall that $\operatorname{deg} \tau_{0}^{R}(M ; z)=\operatorname{dim} M$. Our main result, contained in the theorem below, is a lower bound on the degree of $\tau_{i}^{R}(M ; z)$ for $i \geq 1$. The upper bound is already in [5, (2)] and is valid even when $\mathfrak{m}$ is substituted by any $\mathfrak{m}$-primary ideal. A convention: The degree of a polynomial $t(z)$ is -1 if and only if $t(z)=0$.

Theorem I. Let $R$ be a local ring with $\operatorname{depth} R \geq 1$, and $M$ a non-zero finite $R$-module. For $i \in \mathbb{N}$, if $\operatorname{projdim}_{R} M \geq i$, then $\operatorname{Tor}_{i}^{R}\left(M, R / \mathfrak{m}^{n+1}\right) \neq 0$ for each integer $n \geq 0$, and

$$
\operatorname{dim} R-1 \geq \operatorname{deg} \tau_{i}^{R}(M ; z) \geq \operatorname{depth} R-1 .
$$

Received by the editors November 18, 2004 and, in revised form, September 22, 2005.

2000 Mathematics Subject Classification. Primary 13D40; Secondary 13D02, 13D07.

Key words and phrases. Hilbert-Samuel functions, growth and vanishing of derived functors.

The first author was partly supported by NSF grant DMS 0442242.

(c)2006 American Mathematical Society Reverts to public domain 28 years from publication 
Specializing to the case where $R$ is Cohen-Macaulay yields the following result; it subsumes [7, (18)], which assumes in addition that $M$ is maximal Cohen-Macaulay.

Corollary II. Let $R$ be a Cohen-Macaulay local ring with $\operatorname{dim} R \geq 1$, and $M a$ non-zero finite $R$-module. For $i \in \mathbb{N}$, if $\operatorname{projdim}_{R} M \geq i$, then

$$
\operatorname{deg} \tau_{i}^{R}(M ; z)=\operatorname{dim} R-1 .
$$

This contrasts drastically with the general situation.

Proposition III. Given non-negative integers $p, q$ with $p \leq q-1$, there exists a local ring $R$ with $\operatorname{depth} R=p+1$ and $\operatorname{dim} R=q$, and a finite maximal CohenMacaulay $R$-module $M$ with $\operatorname{deg} \tau_{1}^{R}(M ; z)=p$.

Therefore, in Corollary [I] it is crucial that $R$ is Cohen-Macaulay. However, we have been unable to find a module $M$ for which the inequalities in Theorem $\square$ are strict. On the other hand, towards the end of Section 2 we provide an example that shows that it is not possible to replace $\mathfrak{m}$ with an arbitrary $\mathfrak{m}$-primary ideal.

The preceding results are proved in Section 2, Our proof of the lower bound in Theorem I, that $\operatorname{deg} \tau_{i}^{R}(M ; z) \geq \operatorname{depth} R-1$, proceeds via induction on $\operatorname{depth} R$. The non-vanishing of Tor is an immediate consequence of the following elementary observation, which appears as Lemma 2.1 in the text:

If $\operatorname{Tor}_{1}^{R}\left(M, R / \mathfrak{m}^{n+1}\right)=0$ for some integer $n \geq 0$, then $\mathfrak{m}^{n} \Omega_{1}^{R}(M)=0$; hence either $M$ is free or depth $R=0$.

In what follows $\Omega_{d}^{R}(M)$ denotes the $d$ th syzygy in the minimal free resolution of $M$. In Theorem 3.1, the remark above is extended to statements concerning the vanishing of $\operatorname{Tor}_{i}^{R}\left(M, N / \mathfrak{m}^{n+1} N\right)$, where $N$ is another finite $R$-module. These are akin to a result of Levin and Vasconcelos [6, (1.1)], stated as Theorem 3.3. Any one of these, applied with $M=k$ and $N=R$, may be used to deduce that if either $\operatorname{projdim}_{R}\left(R / \mathfrak{m}^{n+1}\right)$ or $\operatorname{injdim}_{R}\left(R / \mathfrak{m}^{n+1}\right)$ is finite, then $\mathfrak{m}^{n+1}=0$ or $R$ is regular.

The discussion in the preceding paragraph suggests the question: If there is a finite $R$-module $N$ with $\operatorname{dim}_{R} N \geq 1$ and an integer $n \geq 0$ such that either $\operatorname{projdim}_{R}\left(N / \mathfrak{m}^{n+1} N\right)$ or $\operatorname{injdim}_{R}\left(N / \mathfrak{m}^{n+1} N\right)$ is finite, then is $R$ regular? Note that one cannot expect such a strong conclusion if one drops the restriction on the dimension of $N$. Indeed, over Cohen-Macaulay rings (with dualizing modules) it is easy to construct modules of finite length and finite projective (injective) dimension.

Section 3 focuses on this question. The main result is stated below; $\rho_{R}(N)$ is defined in (1.6), while $\boldsymbol{A}_{R}(N)$ is defined in (3.5). These numbers are bounded above by the polynomial regularity of $N$, and so are finite; see (1.6) and Lemma 3.6 .

Theorem IV. Let $(R, \mathfrak{m}, k)$ be a local ring and let $N$ be a finite $R$-module with $\operatorname{depth}_{R} N \geq 1$ and $\operatorname{injdim}_{R}\left(N / \mathfrak{m}^{n+1} N\right)$ finite for some non-negative integer $n$. If $n \geq \rho_{R}(N)$, then $R$ is a hypersurface; if $n \geq \boldsymbol{A}_{R}(N)$ as well, then $R$ is regular.

This is the content of Theorem 3.8 an analogue for projective dimension is given in Theorem 3.7. Our proof of the result above uses superficial sequences and has a different flavour from that of Theorem 3.7. which is easily deduced from available literature. Superficial sequences and other techniques traditionally used in the study of Hilbert functions play an important part in our arguments in Sections 2 and 3. The relevant definitions and results are recalled in Section 1. 


\section{Preliminaries}

Let $(R, \mathfrak{m}, k)$ be a local ring, with maximal ideal $\mathfrak{m}$ and residue field $k$. Let $M$ be a finite $R$-module. We write $\operatorname{gr}_{\mathfrak{m}}(M)$ for $\bigoplus_{n \geqslant 0} \mathfrak{m}^{n} M / \mathfrak{m}^{n+1} M$ and view it as a graded module over the associated graded ring $\operatorname{gr}_{\mathfrak{m}}(R)$.

1.1. Recall that the Hilbert function of $M$ assigns to each integer $n$ the value $\operatorname{rank}_{k}\left(\mathfrak{m}^{n} M / \mathfrak{m}^{n+1} M\right)$. Its generating series is the Hilbert series of $M$; we denote it $\operatorname{Hilb}_{M}(z)$. This series can be expressed as a rational function of the form

$$
\operatorname{Hilb}_{M}(z)=\frac{h_{M}(z)}{(1-z)^{\operatorname{dim} M}} \quad \text { with } \quad h_{M}(z) \in \mathbb{Z}[z] .
$$

The multiplicity of $M$ is the integer $e(M)=h_{M}(1)$; see [2, (4.6)] for details.

1.2. Let $x$ be an element in $\mathfrak{m}$. When $x$ is non-zero, let $n$ be the largest integer such that $x \in \mathfrak{m}^{n}$, and let $x^{*}$ denote the image of $x$ in $\mathfrak{m}^{n} / \mathfrak{m}^{n+1}$. Set $0^{*}=0$.

The element $x \in \mathfrak{m}$ is superficial for $M$ if there exists a positive integer $c$ with

$$
\left(\mathfrak{m}^{n+1} M:{ }_{M} x\right) \cap \mathfrak{m}^{c} M=\mathfrak{m}^{n} M \text { for all } n \geq c .
$$

The following properties of superficial elements are often invoked in this work.

1.3. Let $M_{1}, \ldots, M_{s}$ be a finite $R$-modules.

(a) If $k$ is infinite, then there exists an element $x$ that is superficial for each $M_{j}$.

(b) If an element $x$ is superficial on $M_{j}$ and $\operatorname{depth}_{R} M_{j} \geq 1$, then $x$ is a non-zero divisor on $M_{j}$ and $\left(\mathfrak{m}^{n+1} M_{j}: M_{j} x\right)=\mathfrak{m}^{n} M_{j}$ for $n \gg 0$.

Indeed, it suffices to verify the desired properties for $M=M_{1} \oplus \cdots \oplus M_{s}$. Now (a) is trivial when $\operatorname{dim} M=0$. When $\operatorname{dim} M \geq 1$, an element $x$ is superficial on $M$ if and only if $x \notin \mathfrak{m}^{2}$ and $x^{*}$ is not in any associated prime ideal of $\operatorname{gr}_{\mathfrak{m}}(M)$, except perhaps $\operatorname{gr}_{\mathfrak{m}}(R)_{\geqslant 1}$. Thus, superficial elements exist if the residue field $k$ is infinite. As for (b), an argument of Sally [9, p. 7] for $M=R$ extends to the general case.

Lead by property (b) above, for each element $x$ superficial on $M$ we set

$$
\rho_{R}(x, M)=\inf \left\{r \mid\left(\mathfrak{m}^{n+1} M:{ }_{M} x\right)=\mathfrak{m}^{n} M \quad \text { for each } n \geq r\right\} .
$$

We provide an upper bound for this number; it involves the following invariant.

1.4. Let $\mathcal{M}$ denote the irrelevant maximal ideal of $\operatorname{gr}_{\mathfrak{m}}(R)$, and $\mathrm{H}_{\mathcal{M}}^{j}\left(\operatorname{gr}_{\mathfrak{m}}(M)\right)$ the $j$ th local cohomology of $\operatorname{gr}_{\mathfrak{m}}(M)$ with respect to $\mathcal{M}$. Set

$$
\operatorname{polyreg}_{R}(M)=\sup \left\{p \mid \mathrm{H}_{\mathcal{M}}^{j}\left(\operatorname{gr}_{\mathfrak{m}}(M)\right)_{p-j} \neq 0 \quad \text { for some } j\right\} \text {. }
$$

This number is called the polynomial regularity of $M$ over $R$; see Şega [10, (1.6)].

Lemma 1.5. If $\operatorname{depth}_{R} M \geq 1$, then $\rho_{R}(x, M) \leq \operatorname{polyreg}_{R}(M)+1$.

Remark 1.6. Thanks to the lemma, the number

$$
\rho_{R}(M)=\sup \left\{\rho_{R}(x, M) \mid x \text { an element superficial on } M\right\}
$$

satisfies the inequality $\rho_{R}(M) \leq \operatorname{polyreg}_{R}(M)+1$, and is in particular finite. This result is subsumed in $\left[8\right.$, where it is proved that $\rho_{R}(x, M)$ is independent of $x$ and 
the bound is improved to $\rho_{R}(x, M) \leq \operatorname{polyreg}_{R}(M)$. So we provide only a Sketch of the proof of Lemma 1.5. One has an equality of formal Laurent series

$$
\sum_{n \geq 0} \ell_{R}\left(M / \mathfrak{m}^{n+1} M\right) z^{n}=\frac{\operatorname{Hilb}_{M}(z)}{(1-z)} ;
$$

this is immediate from (1.1); the formal power series on the left is the HilbertSamuel series of $M$. In particular, there exists an integer $c$ and a polynomial $H S_{M}(z)$ such that $\ell_{R}\left(M / \mathfrak{m}^{n+1} M\right)=H S_{M}(n)$ for $n \geq c$. The least such number $c$ is called the postulation number of $M$; we denote it $\operatorname{post}_{R}(M)$. Set $N=M / x M$. Arguing along the lines of the proof of Elias' result [4, (1.1)], one obtains

$$
\rho_{R}(x, M) \leq \max \left\{\operatorname{post}_{R}(M), \operatorname{post}_{R}(N)\right\}+1 .
$$

Now $\operatorname{post}_{R}(M) \leq \operatorname{polyreg}_{R}(M)$ and $\operatorname{post}_{R}(N) \leq \operatorname{polyreg}_{R}(N)$; this is immediate from [2, (4.4.3)], and the definition of $\operatorname{polyreg}_{R}(-)$ recalled above. Moreover, $x$ is a superficial non-zero divisor on $M$, by (1.3), so $\operatorname{polyreg}_{R}(N) \leq \operatorname{polyreg}_{R}(M)$. Combining the inequalities above yields the desired result.

A standard trick allows one to assume that superficial elements exist.

1.7. Let $R[X]$ be a polynomial ring over $R$ on a finite set $X$ of variables. Set $R^{\prime}=R[X]_{\mathfrak{m}^{\prime}}$, where $\mathfrak{m}^{\prime}=\mathfrak{m} R[X]$ and $M^{\prime}=M \otimes_{R} R^{\prime}$. The ring $R^{\prime}$ is again local, with maximal ideal $\mathfrak{m} R^{\prime}$, which we again denote $\mathfrak{m}^{\prime}$, and residue field $k^{\prime}=k(X)$, the field of rational functions over $k$. The following claims are easily verified:

(a) $\ell_{R} M=\ell_{R^{\prime}} M^{\prime}$.

(b) $\tau_{i}^{R}(M ; z)=\tau_{i}^{R^{\prime}}\left(M^{\prime} ; z\right)$ for each non-negative integer $i$.

(c) $\operatorname{embdim} R=\operatorname{embdim} R^{\prime}, \operatorname{dim} M=\operatorname{dim} M^{\prime}$ and $\operatorname{depth} M=\operatorname{depth} M^{\prime}$.

(d) $\operatorname{inj}_{\operatorname{dim}}\left(M / \mathfrak{m}^{n} M\right)$ is finite if and only if $\operatorname{injdim}_{R^{\prime}}\left(M^{\prime} /\left(\mathfrak{m}^{\prime}\right)^{n} M^{\prime}\right)$ is finite.

\section{GROWTH}

In this section we prove Theorem】from the Introduction. Parts of the arguments are abstracted out in the following lemmas. The one below is extended in Theorem 3.1. the crux of the argument is simple and well illustrated in this special case.

Lemma 2.1. Let $(R, \mathfrak{m}, k)$ be a local ring and $M$ a finite $R$-module. If there is an integer $n \geq 0$ with $\operatorname{Tor}_{1}^{R}\left(M, R / \mathfrak{m}^{n+1}\right)=0$, then $\mathfrak{m}^{n} \Omega_{1}^{R}(M)=0$; hence either $M$ is free or $\operatorname{depth} R=0$.

Proof. Set $L=\Omega_{1}^{R}(M)$ and let $0 \rightarrow L \rightarrow F \rightarrow M \rightarrow 0$ be the exact sequence defining $L$. Applying $-\otimes_{R} R / \mathfrak{m}^{n+1}$ to it yields the exact sequence

$$
0 \rightarrow L / \mathfrak{m}^{n+1} L \rightarrow F / \mathfrak{m}^{n+1} F \rightarrow M / \mathfrak{m}^{n+1} M \rightarrow 0 .
$$

Thus $\mathfrak{m}^{n+1} L=L \cap \mathfrak{m}^{n+1} F$. By construction, $L \subseteq \mathfrak{m} F$, so $\mathfrak{m}^{n} L \subseteq L \cap \mathfrak{m}^{n+1} F$, hence $\mathfrak{m}^{n} L \subseteq \mathfrak{m}^{n+1} L$, so Nakayama's lemma yields $\mathfrak{m}^{n} L=0$. It remains to note that $L$ is a submodule of the free module $F$.

Next we recall the following result, contained in [5, (2)].

2.2. Let $(R, \mathfrak{m}, k)$ be a local ring, $M$ a finite $R$-module, and $i$ a positive integer. The function on the non-negative integers defined by

$$
n \mapsto \ell_{R} \operatorname{Tor}_{i}^{R}\left(M, R / \mathfrak{m}^{n+1} R\right)
$$

is given by a polynomial for $n \gg 0$, and of degree at most $\operatorname{dim} R-1$. 
Remark 2.3. We let $\tau_{i}^{R}(M ; z)$ denote the polynomial that arises in Section 2.2 . Since $\operatorname{Tor}_{i}^{R}(M,-) \cong \operatorname{Tor}_{1}^{R}\left(\Omega_{i-1}^{R}(M),-\right)$ for $i \geq 1$, it follows that

$$
\tau_{i}^{R}(M ; z) \cong \tau_{1}^{R}\left(\Omega_{i-1}^{R}(M) ; z\right) \text { for each } i \geq 1 .
$$

This equality often allows one to obtain results on $\tau_{i}^{R}(M ; z)$ from corresponding statements concerning the case $i=1$.

Remark 2.3 focuses our attention on $\tau_{1}^{R}(M ; z)$; the next result describes its basic properties. Recall our convention that the degree of the polynomial 0 is -1 .

Lemma 2.4. Let $(R, \mathfrak{m}, k)$ be a local ring and $M$ a finite $R$-module.

(1) For each finite $R$-module $N$, one has

$$
\tau_{1}^{R}(M \oplus N ; z)=\tau_{1}^{R}(M ; z)+\tau_{1}^{R}(N ; z),
$$

in particular, $\operatorname{deg} \tau_{1}^{R}(M \oplus N ; z)=\sup \left\{\operatorname{deg} \tau_{1}^{R}(M ; z), \operatorname{deg} \tau_{1}^{R}(N ; z)\right\}$.

(2) If $\operatorname{depth} R \geq 1$ and $M$ is not free, then $\operatorname{deg} \tau_{1}^{R}(M ; z) \geq 0$.

(3) If $0 \rightarrow L \rightarrow M \rightarrow N \rightarrow 0$ is an exact sequence of $R$-modules with $\ell_{R} L$ finite, then $\operatorname{deg} \tau_{1}^{R}(M ; z) \geq \operatorname{deg} \tau_{1}^{R}(N ; z)$.

(4) If $\ell_{R} M$ is finite, then $\operatorname{deg} \tau_{1}^{R}(M ; z)=\operatorname{dim} R-1$.

Proof. The first assertion is immediate from the isomorphisms

$$
\operatorname{Tor}_{1}^{R}\left(M \oplus N, R / \mathfrak{m}^{n+1}\right) \cong \operatorname{Tor}_{1}^{R}\left(M, R / \mathfrak{m}^{n+1}\right) \oplus \operatorname{Tor}_{1}^{R}\left(N, R / \mathfrak{m}^{n+1}\right),
$$

while (2) is contained in Lemma (2.1).

Proof of (3): For each non-negative integer $n$, applying $-\otimes_{R} R / \mathfrak{m}^{n+1}$ to the given exact sequence yields an exact sequence of $R$-modules

$\cdots \rightarrow \operatorname{Tor}_{1}^{R}\left(M, R / \mathfrak{m}^{n+1}\right) \rightarrow \operatorname{Tor}_{1}^{R}\left(N, R / \mathfrak{m}^{n+1}\right) \rightarrow L / \mathfrak{m}^{n+1} L \rightarrow M / \mathfrak{m}^{n+1} M \rightarrow \cdots$.

Since $\ell_{R} L$ is finite, the map $L / \mathfrak{m}^{n+1} L \rightarrow M / \mathfrak{m}^{n+1} M$ is injective for $n \gg 0$. For such $n$, computing lengths in the sequence above yields

$$
\ell_{R} \operatorname{Tor}_{1}^{R}\left(M, R / \mathfrak{m}^{n+1}\right) \geq \ell_{R} \operatorname{Tor}_{1}^{R}\left(N, R / \mathfrak{m}^{n+1}\right) .
$$

This implies the desired result.

Proof of (4): It suffices to prove that $\operatorname{deg} \tau_{1}^{R}(M ; z) \geq \operatorname{dim} R-1$; this is due to (2.2). In view of part (3) above, a standard induction on the length reduces the claim to the case $M=k$. Note that for each non-negative integer $n$ one has an isomorphism of $R$-modules

$$
\operatorname{Tor}_{1}^{R}\left(k, R / \mathfrak{m}^{n+1}\right) \cong\left(\mathfrak{m} \cap \mathfrak{m}^{n+1}\right) / \mathfrak{m}^{n+2}=\mathfrak{m}^{n+1} / \mathfrak{m}^{n+2} .
$$

Thus, the degree of $\tau_{1}^{R}(M ; z)$ equals the degree of the Hilbert polynomial of $R$, that is to say, equals $\operatorname{dim} R-1$. This settles (3).

The proof of Theorem \is an induction on depth $R$, and uses the following.

Lemma 2.5. Let $(R, \mathfrak{m}, k)$ be a local ring and $M$ a finite non-free $R$-module. Let $x \in \mathfrak{m}$ be a superficial non-zero divisor on $R, M$, and $\Omega_{1}^{R}(M)$. Set $S=R / x R$ and $N=M / x M$. For $n \gg 0$, one has

$$
\begin{aligned}
& \tau_{1}^{R}(M ; n)=\tau_{1}^{R}(M ; n-1)+\tau_{1}^{S}(N ; n-1), \\
& \operatorname{deg} \tau_{1}^{R}(M ; z)=\operatorname{deg} \tau_{1}^{S}(N ; z)+1 .
\end{aligned}
$$


Proof. Let $\mathfrak{n}$ be the maximal ideal of $S$. By (1.3), since $x$ is superficial on $R$, for each $n \gg 0$ one has an exact sequence of $R$-modules

$$
0 \rightarrow R / \mathfrak{m}^{n} \stackrel{\kappa}{\rightarrow} R / \mathfrak{m}^{n+1} \rightarrow S / \mathfrak{n}^{n+1} \rightarrow 0,
$$

where $\kappa$ maps the residue class of $a$ in $R / \mathfrak{m}^{n}$ to the residue class of $x a$ in $R / \mathfrak{m}^{n+1}$, for each $a \in R$. Applying $M \otimes_{R}-$ to it yields an exact sequence of $R$-modules

$$
\begin{gathered}
\operatorname{Tor}_{1}^{R}\left(M, R / \mathfrak{m}^{n}\right) \stackrel{\operatorname{Tor}_{1}^{R}(M, \kappa)}{\longrightarrow} \operatorname{Tor}_{1}^{R}\left(M, R / \mathfrak{m}^{n+1}\right) \rightarrow \operatorname{Tor}_{1}^{R}\left(M, S / \mathfrak{n}^{n+1}\right) \\
\quad \rightarrow M / \mathfrak{m}^{n} M \stackrel{M \otimes_{R} \kappa}{\longrightarrow} M / \mathfrak{m}^{n+1} M \rightarrow N / \mathfrak{n}^{n+1} N \rightarrow 0 .
\end{gathered}
$$

The maps $M \otimes_{R} \kappa$ and $\operatorname{Tor}_{1}^{R}(M, \kappa)$ are injective for $n \gg 0$. Indeed, the former is injective by Section 1.3 because $x$ is superficial on $M$. As to the latter, set $L=\Omega_{1}^{R}(M)$ and consider the commutative diagram

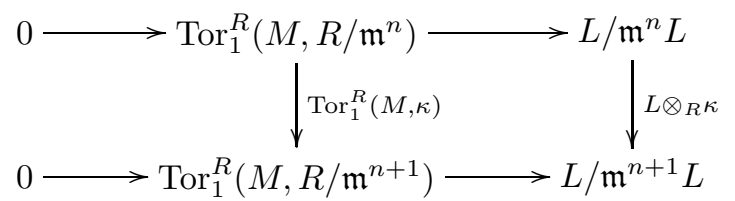

obtained from the exact sequence $0 \rightarrow L \rightarrow F \rightarrow M \rightarrow 0$ defining $L$ by applying $-\otimes_{R} R / \mathfrak{m}^{n}$ and $-\otimes_{R} R / \mathfrak{m}^{n+1}$. By choice, $x$ is superficial on $L$, so for $n \gg 0$ the map $L \otimes_{R} \kappa$ is injective, again by Section 1.3. Thus, $\operatorname{Tor}_{1}^{R}(M, \kappa)$ is also injective.

Returning to the long exact sequence above with the injectivity on hand, one finds for each $n \gg 0$ the following exact sequence of $R$-modules:

$$
0 \rightarrow \operatorname{Tor}_{1}^{R}\left(M, R / \mathfrak{m}^{n}\right) \rightarrow \operatorname{Tor}_{1}^{R}\left(M, R / \mathfrak{m}^{n+1}\right) \rightarrow \operatorname{Tor}_{1}^{R}\left(M, S / \mathfrak{n}^{n+1}\right) \rightarrow 0 .
$$

Now $\operatorname{Tor}_{1}^{R}\left(M, S / \mathfrak{n}^{n+1}\right) \cong \operatorname{Tor}_{1}^{S}\left(N, S / \mathfrak{n}^{n+1}\right)$, as $x$ is both $R$-regular and $M$-regular. This isomorphism and the exact sequence above yield, for $n \gg 0$, an equality

$$
\ell_{R} \operatorname{Tor}_{1}^{R}\left(M, R / \mathfrak{m}^{n+1}\right)=\ell_{R} \operatorname{Tor}_{1}^{R}\left(M, R / \mathfrak{m}^{n}\right)+\ell_{R} \operatorname{Tor}_{1}^{S}\left(N, S / \mathfrak{n}^{n+1}\right) .
$$

Thus, $\tau_{1}^{R}(M ; n)=\tau_{1}^{R}(M ; n-1)+\tau_{1}^{S}(N ; n)$. The hypothesis implies $\Omega_{1}^{R}(M) \neq 0$ and depth $R \geq 1$, so $\operatorname{Tor}_{1}^{R}\left(M, R / \mathfrak{m}^{n+1}\right)$ is non-zero for each $n \geq 0$, by Lemma 2.1. Consequently, the displayed equality yields $\operatorname{deg} \tau_{1}^{R}(M ; z)=\operatorname{deg} \tau_{1}^{S}(N ; z)+1$, even when $\operatorname{Tor}_{i}^{S}\left(N, S / \mathfrak{n}^{n+1}\right)=0$ for $n \gg 0$, that is to say, when $\operatorname{deg} \tau_{1}^{S}(N ; z)=-1$.

2.6. Proof of Theorem I. In view of Remark 2.3, it suffices to verify the theorem for $i=1$; the hypothesis on $M$ is then that it is not free. The first part of the theorem, that $\operatorname{Tor}_{1}^{R}\left(M, R / \mathfrak{m}^{n+1}\right) \neq 0$ for each $n \geq 0$, is immediate from Lemma 2.1. Given Section 2.2, it remains to prove that $\operatorname{deg} \tau_{1}^{R}(M ; z) \geq \operatorname{depth} R-1$.

To establish this we induce on $\operatorname{depth} R$; the base case $\operatorname{depth} R=1$ is easily settled: use Lemma 2.4(2). Assume that the desired inequality has been verified for local rings of depth $d$, for some $d \geq 1$. Let $R$ be a local ring of depth $d+1$ and $M$ a finite, non-free $R$-module.

When $\operatorname{dim}_{R} M=0$, the desired result is provided by Lemma 2.4 (4).

Suppose $\operatorname{dim}_{R} M \geq 1$. By Lemma 2.4(1) we may assume $M$ is indecomposable. Set $L=\left\{m \in M \mid \mathfrak{m}^{s} \cdot m=0\right.$ for some $\left.s \geq 1\right\}$. Since the length of $L$ is finite it suffices to verify the inequality for $M / L$, by Lemma 2.4(3). Now $\operatorname{depth}_{R}(M / L) \geq 1$ and $M / L$ is not free; the last property holds because $M$ is indecomposable and not free. Thus, substituting $M / L$ for $M$ one may assume $\operatorname{depth}_{R} M \geq 1$. 
By Section 1.7. one can extend the residue field of $R$ and ensure that it is infinite, so by Section 1.3 there is an element $x$ that is a superficial non-zero divisor on $R$, $M$, and $\Omega_{1}^{R}(M)$. Hence the local ring $S=R / x R$ has depth $d$, and the $S$-module $N=M / x M$ is not free, since $\operatorname{projdim}_{S} N=\operatorname{projdim}_{R} M$. Lemma 2.5 now provides the equality below:

$$
\operatorname{deg} \tau_{1}^{R}(M ; z)=\operatorname{deg} \tau_{1}^{S}(N ; z)+1 \geq \operatorname{depth} S-1+1=\operatorname{depth} R-1,
$$

while the induction hypothesis yields the inequality.

Next we prepare for the proof of Proposition III

2.7. Let $(S, \mathfrak{n}, k)$ be a local ring and let $L$ be a finitely generated $S$-module. Set $R=S \ltimes L$; thus $R=S \oplus L$, with multiplication given by $(s, l) \cdot\left(s^{\prime}, l^{\prime}\right)=\left(s s^{\prime}, s l^{\prime}+s^{\prime} l\right)$. Evidently, $R$ is a local ring with maximal ideal $\mathfrak{m}=\mathfrak{n} \oplus L$. We view $S$ as an $R$ module via the canonical surjection $R \rightarrow S$. In particular

$$
\operatorname{dim}_{R} S=\operatorname{dim} S \quad \text { and } \quad \operatorname{depth}_{R} S=\operatorname{depth} S .
$$

Note that $\operatorname{dim} R=\operatorname{dim} S$ and $\operatorname{depth} R=\min \left\{\operatorname{depth} S, \operatorname{depth}_{S} L\right\}$, so $R$ is CohenMacaulay if and only if $S$ is Cohen-Macaulay and the $S$-module $L$ is maximal Cohen-Macaulay. The information of interest to us is contained in the following.

Lemma. For each non-negative integer $n$ one has

$$
\ell_{R} \operatorname{Tor}_{1}^{R}\left(S, R / \mathfrak{m}^{n+1}\right)=\operatorname{rank}_{k}\left(\mathfrak{n}^{n} L / \mathfrak{n}^{n+1} L\right) .
$$

In particular, $\operatorname{deg} \tau_{1}^{R}(S ; z)=\operatorname{dim}_{S} L-1$.

Proof. Note that the kernel of the canonical surjection $R \rightarrow S$ is $L$, viewed as an ideal of $R$. This implies the first isomorphism below:

$$
\operatorname{Tor}_{1}^{R}\left(S, R / \mathfrak{m}^{n+1}\right) \cong\left(L \cap \mathfrak{m}^{n+1}\right) / \mathfrak{m}^{n+1} L \cong \mathfrak{n}^{n} L / \mathfrak{n}^{n+1} L .
$$

The second equality holds because $\mathfrak{m}=\mathfrak{n} \oplus L$ and $L^{2}=0$. Now compute lengths.

Proof of Proposition 【II] Let $k$ be a field, $S=k\left[\left[x_{1}, \ldots, x_{q}\right]\right]$ the ring of formal power series in variables $x_{1}, \ldots, x_{q}$, and let $L=S /\left(x_{p+2}, \ldots, x_{q}\right)$. The local ring $S$ is Cohen-Macaulay with $\operatorname{dim} S=q$, and $L$ is a finite Cohen-Macaulay $S$-module with $\operatorname{dim}_{S} L=p+1$. Set $R=S \ltimes L$, and let $M=S$, viewed as an $R$-module via the canonical surjection $R \rightarrow S$. By (2.7), one has $\operatorname{depth} R=p+1, \operatorname{dim} R=q$, $\operatorname{deg} \tau_{1}^{R}(M ; z)=p$, and $M$ is a maximal Cohen-Macaulay $R$-module.

Another interpretation of this proposition is as follows: for any integer $s \geq 1$ there exists a local ring $R$ and a maximal Cohen-Macaulay $R$-module $M$ such that $\operatorname{dim} R-\operatorname{deg} \tau_{1}^{R}(M ; z)=s$. Thus, the inequality in [13, (4.a)] is strict, in general.

It is also interesting to study the growth of the function defined by

$$
n \mapsto \ell_{R} \operatorname{Tor}_{i}^{R}\left(M, R / I^{n+1}\right)
$$

for an arbitrary $\mathfrak{m}$-primary ideal $I$. In this case as well the function is given by a polynomial for $n \gg 0$, and its degree is at most $\operatorname{dim} R-1$, by [5, (2)]. However, the upper bound may be strict even if $R$ is Cohen-Macaulay: When the residue field of such an $R$ is infinite and $M$ is a non-free maximal Cohen-Macaulay module, there are $\mathfrak{m}$-primary ideals $I$ such that $\operatorname{Tor}_{1}^{R}\left(M, R / I^{n+1}\right)=0$ for $n \gg 0$; see [7, (20)]. 


\section{Finite homologicAl Dimensions}

The results in this section are motivated by the

Question. Let $R$ be a local ring and $N$ a finite $R$-module such that, for some integer $n \geq 0$, the $R$-module $N / \mathfrak{m}^{n+1} N$ has either finite projective dimension or finite injective dimension. If $\operatorname{dim}_{R} N \geq 1$, then is $R$ regular?

These questions are suggested by Theorem 3.1 below and its corollaries. As noted in the Introduction, some restriction on $\operatorname{dim}_{R} N$ or on $\operatorname{depth}_{R} N$ is crucial. We begin with the result below; with $N=R$ and $n=1$ it captures Lemma 2.1.

Theorem 3.1. Let $(R, \mathfrak{m}, k)$ be a local ring, and let $M$ and $N$ be finite $R$-modules. If there exist non-negative integers $i$ and $n$ such that

$$
\operatorname{Tor}_{i}^{R}(M, N)=0=\operatorname{Tor}_{i}^{R}\left(M, N / \mathfrak{m}^{n+1} N\right),
$$

then $\mathfrak{m}^{n}\left(\Omega_{i}^{R}(M) \otimes_{R} N\right)=0$ and either $\operatorname{depth}_{R} N=0$ or $\operatorname{projdim}_{R} M \leq i-1$.

With stronger hypotheses, we are able to arrive at a better conclusion.

Theorem 3.2. Let $(R, \mathfrak{m}, k)$ be a local ring, and let $M$ and $N$ be finite $R$-modules. If there exist non-negative integers $i$ and $n$ such that

$$
\operatorname{Tor}_{i}^{R}\left(M, N / \mathfrak{m}^{n+1} N\right)=0=\operatorname{Tor}_{i}^{R}\left(M, \mathfrak{m}^{n+1} N\right),
$$

then either $\mathfrak{m}^{n+1} N=0$ or $\operatorname{projdim}_{R} M \leq i-1$.

These theorems are akin to the one below, contained in [6, Lemma, p. 316].

Theorem 3.3. Let $(R, \mathfrak{m}, k)$ be a local ring, and let $M$ and $N$ be finite $R$-modules. If there exist non-negative integers $i$ and $n$ such that

$$
\operatorname{Tor}_{i}^{R}\left(M, \mathfrak{m}^{n+1} N\right)=0=\operatorname{Tor}_{i+1}^{R}\left(M, \mathfrak{m}^{n+1} N\right)
$$

then either $\mathfrak{m}^{n+1} N=0$ or $\operatorname{projdim}_{R} M \leq i-1$.

Evidently, Theorems 3.1, 3.2, and 3.3 are closely related: Consider the exact sequence of $R$-modules $0 \rightarrow \mathfrak{m}^{n+1} N \rightarrow N \rightarrow N / \mathfrak{m}^{n+1} N \rightarrow 0$. However, we have been unable to deduce one from the other, nor find a useful common generalization. We should like to note that statements analogous to the ones above with $\operatorname{Tor}_{*}^{R}(M,-)$ replaced by $\operatorname{Ext}_{R}^{*}(M,-)$ also hold. Now we turn to the

Proof of Theorems 3.1 and 3.2. We may assume that $M$ and $N$ are non-zero. Thus, $\operatorname{Tor}_{0}^{R}(M, N)=M \otimes_{R} N \neq 0$, so $i \geq 1$, and

$$
\operatorname{Tor}_{i}^{R}(M,-) \cong \operatorname{Tor}_{1}^{R}\left(\Omega_{i-1}^{R}(M),-\right) \quad \text { and } \quad \operatorname{projdim}_{R} M=\operatorname{projdim}_{R} \Omega_{i}^{R}(M)+i \text {. }
$$

Hence, replacing $M$ with $\Omega_{i-1}^{R}(M)$ one may assume that $i=1$. Set $L=\Omega_{1}^{R}(M)$ and $F=R^{\nu(M)}$, and consider the exact sequence of $R$-modules

$$
0 \rightarrow L \rightarrow F \rightarrow M \rightarrow 0
$$


Claim: There is a commutative diagram of homomorphisms of $R$-modules:

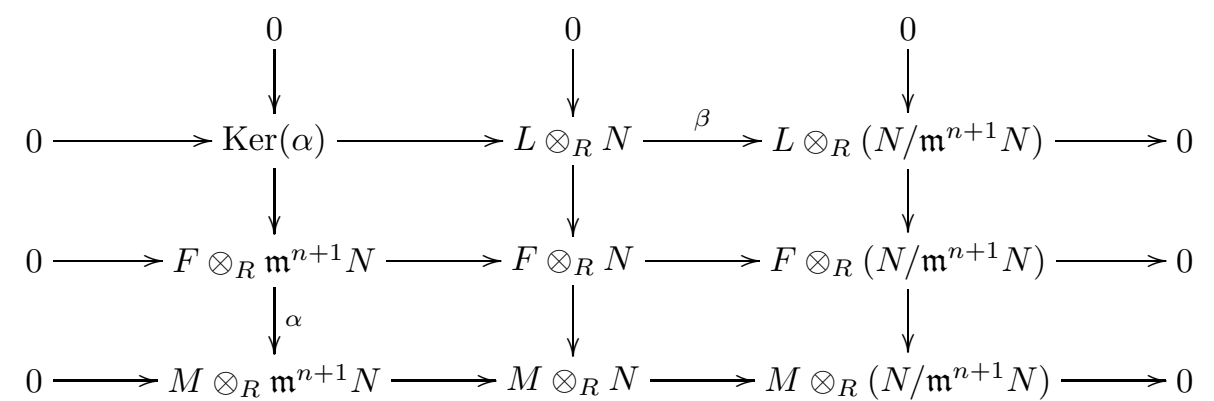

where the sequences in the rows and columns are exact, and, under the hypotheses of Theorem 3.2. also $\operatorname{Ker}(\alpha)=L \otimes_{R} \mathfrak{m}^{n+1} N$.

Indeed, tensoring $(\dagger)$ with the exact sequence

$$
0 \rightarrow \mathfrak{m}^{n+1} N \rightarrow N \rightarrow N / \mathfrak{m}^{n+1} N \rightarrow 0
$$

yields the desired commutative diagram. Now, under either sets of hypotheses, $\operatorname{Tor}_{1}^{R}\left(M, N / \mathfrak{m}^{n+1} N\right)=0$, so the last row and the rightmost column are also exact. The second row is exact because the $R$-module $F$ is free, while the second column is exact because $\operatorname{Tor}_{1}^{R}(M, N)=0$; this is part of the hypotheses in Theorem 3.1] and follows from that of Theorem 3.2 and the exact sequence $(\ddagger)$ above. The exactness of the last two rows and the surjectivity of $\alpha$ imply, by the snake lemma, that the first row is exact. Finally, in Case (3.2), the leftmost column is exact and $\operatorname{Ker}(\alpha)=L \otimes_{R} \mathfrak{m}^{n+1} N$.

To finish the proof, we consider the two cases separately. In either case we may suppose that $\operatorname{projdim}_{R} M \geq 1$, that is to say, $M$ is not free. Thus, $L \neq 0$.

Case (3.1). Viewing $F \otimes_{R} \mathfrak{m}^{n+1} N$ and $L \otimes_{R} N$ as submodules of $F \otimes_{R} N$, one has $\operatorname{Ker}(\alpha)=\operatorname{Ker}(\beta)$. This explains the second equality below:

$$
\left(L \otimes_{R} N\right) \cap \mathfrak{m}^{n+1}\left(F \otimes_{R} N\right)=\left(L \otimes_{R} N\right) \cap\left(F \otimes_{R} \mathfrak{m}^{n+1} N\right)=\mathfrak{m}^{n+1}\left(L \otimes_{R} N\right),
$$

while the first is due to the $R$-linearity of the tensor product. On the other hand, $L \subseteq \mathfrak{m} F$, so $L \otimes_{R} N \subseteq \mathfrak{m}\left(F \otimes_{R} N\right)$, and hence $\mathfrak{m}^{n}\left(L \otimes_{R} N\right) \subseteq \mathfrak{m}^{n+1}\left(F \otimes_{R} N\right)$. Combining this inclusion with the equality above yields

$$
\mathfrak{m}^{n}\left(L \otimes_{R} N\right) \subseteq\left(L \otimes_{R} N\right) \cap \mathfrak{m}^{n+1}\left(F \otimes_{R} N\right)=\mathfrak{m}^{n+1}\left(L \otimes_{R} N\right) .
$$

Thus $\mathfrak{m}^{n}\left(L \otimes_{R} N\right)=0$, by Nakayama's lemma. This is the first part of the desired result. As to the second, since $L$ is non-zero, $L \otimes_{R} N$ is non-zero as well. Since $\left(L \otimes_{R} N\right) \subseteq\left(F \otimes_{R} N\right)$, by the diagram above, and $\mathfrak{m}^{n}\left(L \otimes_{R} N\right)=0$, we deduce that $\operatorname{depth}\left(F \otimes_{R} N\right)=0$. However, $F \otimes_{R} N \cong N^{\nu(M)}$, so depth $N=0$, as claimed.

Case (3.2). Recall that $\operatorname{Ker}(\alpha)=L \otimes_{R} \mathfrak{m}^{n+1} N$. Viewing the modules in the top left-hand square of the diagram above as submodules of $F \otimes_{R} N$, one has that $L \otimes_{R} \mathfrak{m}^{n+1} N=\mathfrak{m}^{n+1}\left(L \otimes_{R} N\right)=0$, where the second equality holds because $\mathfrak{m}^{n}\left(L \otimes_{R} N\right)=0$, by Case (3.1). However, $L \neq 0$, so $\mathfrak{m}^{n+1} N=0$, as desired.

Here is one consequence of Theorem 3.2 ,

Corollary 3.4. Let $(R, \mathfrak{m}, k)$ be a local ring, $N$ a finite $R$-module with $\mathfrak{m}^{n+1} N \neq 0$ and $\operatorname{projdim}_{R}\left(N / \mathfrak{m}^{n+1} N\right)$ finite for an integer $n \geq 0$. For each finite $R$-module $M$ with $\operatorname{projdim}_{R} M=\infty$, one has $\operatorname{Tor}_{i}^{R}(M, N) \neq 0$ for each integer $i \geq \operatorname{depth} R+1$. 
Proof. Fix an integer $i \geq \operatorname{depth} R+1$. The Auslander-Buchsbaum equality implies $\operatorname{projdim}_{R}\left(N / \mathfrak{m}^{n+1} N\right)=\operatorname{depth} R$, so $\operatorname{Tor}_{j}^{R}\left(M, N / \mathfrak{m}^{n+1} N\right)=0$ for $j=i, i+1$. Thus, for each $R$-module $M$ the exact sequence $0 \rightarrow \mathfrak{m}^{n+1} N \rightarrow N \rightarrow N / \mathfrak{m}^{n+1} N \rightarrow 0$ yields $\operatorname{Tor}_{i}^{R}\left(M, \mathfrak{m}^{n+1} N\right) \cong \operatorname{Tor}_{i}^{R}(M, N)$. Therefore, the desired result follows from (the contrapositive of) Theorem 3.2 .

Next we introduce some invariants used to describe other results in this section.

3.5. Let $N$ be a finite $R$-module. For each non-negative integer $n$, let $\pi^{n}$ denote the canonical surjection $N \rightarrow N / \mathfrak{m}^{n+1} N$. We define the Avramov index of $N$ to be

$$
\boldsymbol{A}_{R}(N)=\inf \left\{n \geq 0 \mid \operatorname{Tor}^{R}\left(k, \pi^{n}\right) \quad \text { is injective }\right\} .
$$

This definition is motivated by a result of Avramov [1, (A.4)], which implies that $\boldsymbol{A}_{R}(N)$ is finite. Şega [10, (5.1)] has introduced the Avramov index of a local ring $R$ to the least integer $n$ such that the map $\operatorname{Tor}^{R}(k, k) \rightarrow \operatorname{Tor}^{R / \mathfrak{m}^{n+1}}(k, k)$, induced by the canonical homomorphism of rings $R \rightarrow R / \mathfrak{m}^{n+1}$, is injective. It is not hard to prove that $\boldsymbol{A}_{R}(\mathfrak{m})$ is less than or equal to the Avramov index of the ring $R$; we do not know if equality holds.

The Levin index of $N$ (see [10, (3.1)]) is the least integer $n$ for which the map $\operatorname{Tor}^{R}\left(k, \mathfrak{m}^{s} N\right) \rightarrow \operatorname{Tor}^{R}\left(k, \mathfrak{m}^{s-1} N\right)$, induced by $\mathfrak{m}^{s} N \subseteq \mathfrak{m}^{s-1} N$, is zero for $s \geq n$. 1.4

The invariant polyreg $_{R}(N)$ appearing in the lemma below is defined in Section

Lemma 3.6. Let $R$ be a local ring. For each finite $R$-module $N$, one has

$$
\boldsymbol{A}_{R}(N) \leq \boldsymbol{L}_{R}(N)-1 \leq \operatorname{polyreg}_{R}(N) .
$$

Proof. Applying $k \otimes_{R}$ - to the exact sequence $0 \rightarrow \mathfrak{m}^{n+1} N \rightarrow N \rightarrow N / \mathfrak{m}^{n+1} N \rightarrow 0$ yields the long exact sequence of $R$-modules

$$
\cdots \rightarrow \operatorname{Tor}_{i}^{R}\left(k, \mathfrak{m}^{n+1} N\right) \rightarrow \operatorname{Tor}_{i}^{R}(k, N) \stackrel{\operatorname{Tor}_{i}^{R}\left(k, \pi^{n}\right)}{\longrightarrow} \operatorname{Tor}_{i}^{R}\left(k, N / \mathfrak{m}^{n+1} N\right) \rightarrow \cdots .
$$

Thus, $\operatorname{Tor}^{R}\left(k, \pi^{n}\right)$ is injective if and only if the map $\operatorname{Tor}^{R}\left(k, \mathfrak{m}^{n+1} N\right) \rightarrow \operatorname{Tor}^{R}(k, N)$ is zero. Since this last map factors through $\operatorname{Tor}^{R}\left(k, \mathfrak{m}^{n} N\right)$, one obtains that if $\operatorname{Tor}^{R}\left(k, \mathfrak{m}^{n+1} N\right) \rightarrow \operatorname{Tor}^{R}\left(k, \mathfrak{m}^{n} N\right)$ is zero, then $\operatorname{Tor}^{R}\left(k, \pi^{n}\right)$ is injective as well. Thus, $\boldsymbol{A}_{R}(N) \leq \boldsymbol{L}_{R}(N)-1$. The remaining inequality is contained in [10, (3.3)].

The next result is easily deduced from available literature, but there is no convenient reference; in any case, it is worth stating it in terms of the Avramov index.

Theorem 3.7. Let $(R, \mathfrak{m}, k)$ be a local ring. If there is a finite $R$-module $N$ with $\operatorname{dim}_{R} N \geq 1$ and $\operatorname{projdim}_{R}\left(N / \mathfrak{m}^{n+1} N\right)$ finite for an $n \geq \boldsymbol{A}_{R}(N)$, then $R$ is regular.

Proof. The condition $n \geq \boldsymbol{A}_{R}(N)$ ensures the injectivity of the map

$$
\operatorname{Tor}^{R}\left(k, \pi^{n}\right): \operatorname{Tor}^{R}(k, N) \longrightarrow \operatorname{Tor}^{R}\left(k, N / \mathfrak{m}^{n+1} N\right)
$$

Therefore, since $\operatorname{projdim}_{R}\left(N / \mathfrak{m}^{n+1} N\right)$ is finite, $\operatorname{Tor}_{i}^{R}(k, N)=0$ for $i \gg 0$, so that $\operatorname{projdim}_{R}(N)$ is also finite. If $\operatorname{depth}_{R} N \geq 1$, then at this point Corollary 3.4 applied with $M=k$ would entail $\operatorname{projdim}_{R} k$ finite, and hence that $R$ is regular. In any case, from the exact sequence

$$
0 \rightarrow \mathfrak{m}^{n+1} N \rightarrow N \rightarrow N / \mathfrak{m}^{n+1} N \rightarrow 0,
$$

one deduces that $\operatorname{projdim}_{R}\left(\mathfrak{m}^{n+1} N\right)$ is finite. Since $\operatorname{dim}_{R} N \geq 1$, Nakayama's lemma implies $\mathfrak{m}^{n+1} N \neq 0$. It now follows from [6, (1.1)] that $R$ is regular. 
The preceding theorem has an analogue for injective dimensions, where, instead of the Avramov index, the invariant of interest is the least integer $n$ such that the canonical homomorphism $\operatorname{Ext}_{R}(k, N) \rightarrow \operatorname{Ext}_{R}\left(k, N / \mathfrak{m}^{n+1} N\right)$ is injective. We take a different route in the ensuing result; the invariant $\rho_{R}(N)$ that appears here was introduced in Remark 1.6. As noted there, it is bounded above by $\operatorname{polyreg}_{R}(N)$. Recall that $R$ is said to be a hypersurface if embdim $R-\operatorname{depth} R \leq 1$.

Theorem 3.8. Let $(R, \mathfrak{m}, k)$ be a local ring and let $N$ be a finite $R$-module with $\operatorname{depth}_{R} N \geq 1$ and $\operatorname{injdim}_{R}\left(N / \mathfrak{m}^{n+1} N\right)$ finite for some non-negative integer $n$.

If $n \geq \rho_{R}(N)$, then $R$ is a hypersurface; if $n \geq \boldsymbol{A}_{R}(N)$ as well, then $R$ is regular.

Proof. Let $L$ be an $R$-module; we set $s_{i}(L)=\ell_{R} \operatorname{Hom}_{R}\left(L, N / \mathfrak{m}^{i+1} N\right)$ and write $\nu_{R}(L)$ for the minimal number of generators $L$. First we establish the claims below.

Claim. One has $s_{n}(L) \geq s_{n-1}(L)$.

Indeed, Section 1.7 allows one to enlarge the residue field and assume that the field $k$ is infinite. Thus, there is an $x$ in $\mathfrak{m}$ superficial on $N$, so for $n \geq \rho(N)$ multiplication by $x$ yields an exact sequence of $R$-modules $0 \longrightarrow N / \mathfrak{m}^{n} N \rightarrow N / \mathfrak{m}^{n+1} N$, and this gives the exact sequence below, which settles the claim:

$$
0 \longrightarrow \operatorname{Hom}_{R}\left(L, N / \mathfrak{m}^{n} N\right) \longrightarrow \operatorname{Hom}_{R}\left(L, N / \mathfrak{m}^{n+1} N\right) .
$$

Claim. If $\operatorname{Ext}_{R}^{1}\left(L, N / \mathfrak{m}^{n+1} N\right)=0$, then $\nu_{R}(L) \geq \nu_{R}\left(\Omega_{1}^{R}(L)\right)$.

Indeed, the exact sequence of $R$-modules

$$
0 \longrightarrow \mathfrak{m}^{n} N / \mathfrak{m}^{n+1} N \longrightarrow N / \mathfrak{m}^{n+1} N \longrightarrow N / \mathfrak{m}^{n} N \longrightarrow 0
$$

induces an exact sequence

$$
\begin{aligned}
0 \longrightarrow \operatorname{Hom}_{R}\left(L, \mathfrak{m}^{n} N / \mathfrak{m}^{n+1} N\right) \longrightarrow \operatorname{Hom}_{R}\left(L, N / \mathfrak{m}^{n+1} N\right) \longrightarrow \operatorname{Hom}_{R}\left(L, N / \mathfrak{m}^{n} N\right) \\
\\
\longrightarrow \operatorname{Ext}_{R}^{1}\left(L, \mathfrak{m}^{n} N / \mathfrak{m}^{n+1} N\right) \longrightarrow \operatorname{Ext}_{R}^{1}\left(L, N / \mathfrak{m}^{n+1} N\right)=0 .
\end{aligned}
$$

Note that $\operatorname{Ext}_{R}^{1}\left(L, \mathfrak{m}^{n} N / \mathfrak{m}^{n+1} N\right) \cong \operatorname{Hom}_{R}\left(\Omega_{1}^{R}(L), \mathfrak{m}^{n} N / \mathfrak{m}^{n+1} N\right)$, so computing lengths one obtains from the exact sequences above an equality

$$
h \cdot \nu_{R}(L)-h \cdot \nu_{R}\left(\Omega_{1}^{R}(L)\right)=s_{n}(L)-s_{n-1}(L),
$$

where $h=\operatorname{rank}_{k}\left(\mathfrak{m}^{n} N / \mathfrak{m}^{n+1} N\right)$. Since $\operatorname{dim}_{R} N \geq 1$ one has $h \geq 1$, so the equality above and the preceding claim imply that $\nu_{R}(L) \geq \nu_{R}\left(\Omega_{1}^{R}(L)\right)$, as desired.

Consider the case when $d=\operatorname{injdim}_{R}\left(N / \mathfrak{m}^{n+1} N\right)$ is finite. For $i \geq d+1$, one has

$$
\operatorname{Ext}_{R}^{1}\left(\Omega_{i}^{R}(k), N / \mathfrak{m}^{n+1} N\right)=\operatorname{Ext}_{R}^{i+1}\left(k, N / \mathfrak{m}^{n+1} N\right)=0 .
$$

Given this, the preceding claim yields $\nu_{R}\left(\Omega_{i}^{R}(k)\right) \geq \nu_{R}\left(\Omega_{i+1}^{R}(k)\right)$. Thus, the Betti numbers of $k$ are bounded, so $R$ is a hypersurface; see Tate [12, Theorem 8]. In particular, $R$ is a hypersurface, and hence Gorenstein, so $\operatorname{projdim}_{R}\left(N / \mathfrak{m}^{n+1} N\right)$ is finite. As $\operatorname{depth}_{R} N \geq 1$, when $n \geq \boldsymbol{A}_{R}(N)$ Theorem 3.7 implies $R$ is regular.

This last result leads us to modules of finite length and finite projective dimension over hypersurfaces. In this context one has the following well-known result; see, for example, Ding [3, (1.5) and (3.3)].

Proposition 3.9. Let $(R, \mathfrak{m}, k)$ be a hypersurface and $M$ a non-zero finite $R$ module. If $\mathfrak{m}^{e(R)-1} M=0$, then $\operatorname{projdim}_{R} M=\infty=\operatorname{injdim}_{R} M$. 
Proof. It is elementary to reduce to the case where $R$ and $M$ are m-adically complete. Now, Cohen's structure theorem provides a surjective homomorphism $(Q, \mathfrak{q}, k) \rightarrow R$ of local rings with $Q$ regular and $\operatorname{embdim} Q=\operatorname{embdim} R$. Since $R$ is a hypersurface, $R=Q /(r)$; it is not hard to verify that $r \in \mathfrak{q}^{e(R)}$. Note that $\mathfrak{q}^{e(R)-1} M=0$, so $r \in \mathfrak{q} \operatorname{Ann}_{Q}(M)$, where $\operatorname{Ann}_{Q}(M)$ denotes the annihilator of $M$ viewed as a module over $Q$. A result of Shamash [11, Corollary 1] now implies that $\operatorname{projdim}_{R} M$ is infinite. Since $R$ is a Gorenstein, injdim $R$ is also infinite.

From Theorem 3.8 and Proposition 3.9 one obtains the following corollary. A hypersurface $R$ that is not regular is a singular hypersurface; thus, $R$ is a singular hypersurface when embdim $R-\operatorname{depth} R=1$.

Corollary 3.10. Let $R$ be a singular hypersurface. Let $N$ be a finite $R$-module with $\operatorname{depth}_{R} N \geq 1$ and set $d=\sup \left\{\rho_{R}(N), \boldsymbol{A}_{R}(N)\right\}$. For each integer $n \geq 0$ with $n \notin[e(R)-1, d-1]$, one has $\operatorname{projdim}_{R}\left(N / \mathfrak{m}^{n+1} N\right)=\infty=\operatorname{injdim}_{R}\left(N / \mathfrak{m}^{n+1} N\right)$.

We end with a question: Does the conclusion of the corollary hold for each $n \geq 0$ ?

\section{ACKNOWLEDGMENTS}

It is a pleasure to thank Lucho Avramov, Shiro Goto, Janet Striuli, and Emanoil Theodorescu for useful conversations regarding this article.

\section{REFERENCES}

[1] L. Avramov, Small homomorphisms of local rings, J. Algebra 50 (1978), 400-453. MR0485906 (81i:13009)

[2] W. Bruns and J. Herzog, Cohen-Macaulay rings, vol. 39, Cambridge Stud. Adv. Math., Cambridge Univ. Press, 1998. MR.1251956 (95h:13020)

[3] S. Ding, Cohen-Macaulay approximations and multiplicity, J. Algebra 153 (1992), 271-288. MR.1198202 (94c:13024)

[4] J. Elias, On the computation of Ratliff-Rush closures, J. Symb. Comput. 37 (2004), 717-725. MR2095368 (2005j:13022)

[5] V. Kodiyalam, Homological invariants of powers of an ideal, Proc. Amer. Math. Soc 118 (1993), 757-764. MR.1156471 (93i:13022)

[6] G. Levin and W. V. Vasconcelos, Homological dimensions and Macaulay rings, Pacific J. Math. 25 (1968), 315-323. MR0230715 (37:6275)

[7] T. J. Puthenpurakal, Hilbert coefficients of a Cohen-Macaulay module, J. Algebra 264 (2003), 82-97. MR 1980687 (2004c:13015)

[8] _ Ratliff-Rush filtration, regularity and depth of higher associated grade modules, Part I, J. Pure App. Algebra, to appear.

[9] J. D. Sally, Number of generators of ideals in local rings, Lect. Notes Pure Appl. Math., vol. 35, M. Dekker, 1978. MR0485852 (58:5654)

[10] L. M. Şega, Homological properties of powers of the maximal ideal of a local ring, J. Algebra 241 (2001), 827-858. MR.1843329 (2002k:13026)

[11] J. Shamash, The Poincaré series of a local ring, J. Algebra 12 (1969), 453-470. MR0241411 $(39: 2751)$

[12] J. Tate, Homology of Noetherian rings and local rings, Illinois J. Math 1 (1957), 14-25. MR0086072 (19:119b)

[13] E. Theodorescu, Derived functors and Hilbert polynomials, Math. Proc. Camb. Phil. Soc. 132 (2002), 75-88. MR.1866325 (2002j:13018)

Department of Mathematics, University of Nebraska, Lincoln, Nebraska 68588

E-mail address: iyengar@math.unl.edu

Department of Mathematics, IIt Bombay, Powai, Mumbai 400 076, India

E-mail address: tputhen@math.iitb.ac.in 